\title{
Suspended sediment load in northwestern South America (Colombia): A new view on variability and fluxes into the Caribbean Sea
}

\author{
Juan Camilo Restrepo López, Andrés F.Orejarena R, Ana Carolina Torregroza
} Espinosa

\begin{abstract}
Monthly averaged suspended sediment load data from seven rivers in northern Colombia (Caribbean alluvial plain) draining into the Caribbean Sea were analyzed to quantify magnitudes, estimate long-term trends, and evaluate variability patterns of suspended sediment load. Collectively these rivers deliver an average of around $146.3 \times 106 \mathrm{t} \mathrm{yr}-1$ of suspended sediments to the Colombian Caribbean coast. The largest sediment supply is provided by the Magdalena River, with a mean suspended sediment load of $142.6 \times 106 \mathrm{tyr}-1$, or $38 \%$ of the total fluvial discharge estimated for the whole Caribbean littoral zone. Between 2000 and 2010, the annual suspended sediment load of these rivers increased by as much as $36 \%$. Wavelet spectral analyses identified periods of intense variability between 1987-1990 and 1994-2002, where major oscillation processes appeared simultaneously. The semiannual, annual and quasi-decadal bands are the main factors controlling suspended sediment load variability in fluvial systems, whereas the quasi-biennial and interannual bands constitute second-order sources of variability. The climatic and oceanographic drivers of the oscillations identified through wavelet spectral analyses define a signal of medium-long-term variability for the suspended sediment load, while the physiographic and environmental characteristics of the basins determine their ability to magnify, attenuate or modify this signal.
\end{abstract}

\section{Keywords}

Suspended Sediment Load, Caribbean Sea, Hydrologic Variability, Wavelet Spectral Analyses 